\title{
COIN TOSSING AND SUM SETS
}

\section{GAVIN BROWN and JOHN H. WILLIAMSON}

(Received 6 November 1985)

Communicated by W. Moran

\begin{abstract}
We consider the distribution $\mu$ of numbers whose binary digits are generated from infinitely many tosses of a biased coin. It is shown that, if $E$ has positive $\mu$ measure, then some $n$-fold sum of $E$ with itself must contain an interval. This contrasts with the known result that all convolution powers of $\mu$ are singular.
\end{abstract}

1980 Mathematics subject classification (Amer. Math. Soc.): primary 60 B 15; secondary 43 A 05.

\section{Introduction}

Let $X$ be a random variable in the unit interval the digits of whose binary expansion are determined by tossing a biased coin ( 0 with probability $p, 1$ with probability $1-p, p \neq 0,1 / 2,1)$. Then it is well known that every $n$-fold sum of independent copies of $X$ has a purely singular distribution: for a proof, note that the characteristic function of $X$ does not vanish at infinity. By way of counterpoint we show here that if $E$ is any Borel set within which there is a positive probability of locating $X$, then some $n$-fold sum of $E$ must contain an interval.

Our result is perhaps the most natural probabilistic way to exhibit a phenomenon previously discussed in the context of Banach algebras of measures [2], [3]. Following [3] we call a measure $\mu$ basic provided that

$$
\mu(E)>0 \Rightarrow \mathrm{Gp}(E)=\mathbb{R},
$$

(c) 1987 Australian Mathematical Society $0263-6115 / 87$ \$A2.00 +0.00 
where $\mathrm{Gp}(E)$ is the subgroup of the additive reals generated by the elements of $E$, Lebesgue measure is obviously basic, but the algebraic property of interest is the existence of basic measures all of whose convolution powers are singular. Cantor measure $\mu_{C}$ (or, more accurately, Lebesgue's singular measure on Cantor's middle third set!) was shown in [2] to be of this type, the case of (most) Riesz products was covered in [3], and the result is extended here to coin-tossing distributions.

We follow the pattern of proof of [2] where the inequality

$$
\lambda(E+F) \geqslant \mu_{C}(E)^{\alpha} \mu_{C}(F)^{\alpha},
$$

( $\lambda$ Lebesgue measure, $\mu_{C}$ Cantor measure, $\alpha=\log 3 / \log 4$ ) was established. Our main theorem gives analogous inequalities for $\boldsymbol{n}$-fold sums involving the coin-tossing distributions. Related inequalities for different classes of measures can be found in [1], [5].

The work reported here was started while the first-named author visited Heriot-Watt University partially supported by SERC.

\section{Main result}

For $0<p<1$, let $\mu_{p}$ denote the infinite convolution

$$
\mu_{p}=\underset{n=1}{*}\left(p \delta_{0}+(1-p) \delta_{2^{-n}}\right),
$$

where $\delta_{x}$ denotes the positive unit mass at $x$. Thus $\mu_{p}$ is the distribution of the random variable $X$ described in the introduction. Let $\lambda$ denote Lebesgue measure on the real line (so that $\lambda$ restricted to $[0,1]$ is $\mu_{1 / 2}$ ).

THEOREM 1. Let $a=[\max (p, 1-p)]^{-1}$. Suppose that $a \geqslant 2^{1 / n}$ and let $\alpha=\alpha_{n}$ $=\log 2 / n \log a$. Suppose that $E_{1}, E_{2}, \ldots, E_{n}$ are Borel subsets of $\mathbb{R}$. Then

$$
\lambda\left(E_{1}+E_{2}+\cdots+E_{n}\right) \geqslant \mu_{p}\left(E_{1}\right)^{\alpha} \mu_{p}\left(E_{2}\right)^{\alpha} \cdots \mu_{p}\left(E_{n}\right)^{\alpha} \text {. }
$$

We will show, in the next section, how to reduce the proof of Theorem 1 to a purely combinatorial result which will be established in Section 4 . For now, let us show how the corollary follows from the theorem. In fact, given $p(0<p<1)$, we have $a \geqslant 1$, and hence there exists a positive integer $n$ such that $a \geqslant 2^{1 / n}$. Suppose now that $E$ is a Borel set of positive $\mu_{p}$-measure and apply the theorem with $E_{1}=E_{2}=\cdots=E_{n}=E$. This shows that the $n$-fold sum, $(n) E$, of $E$ with itself has positive $\lambda$-measure. By a classical theorem of Steinhaus (cf. [4], page $143)$ the sum of $(n) E$ with itself must contain an open interval. If is now clear that the group generated by $E$ covers the entire line, in other words, that $\mu_{p}$ is basic. 
REMARK. The key property in the preceding argument is the fact that some $n$-fold sum of every set of positive $\mu_{p}$-measure has positive Lebesgue measure. It is appropriate to note the extraordinary fact (cf. [2]) that there exist basic measures supported by (closed) null sets all of whose $n$-fold sums are Lebesgue null.

\section{Reduction step}

Our object in this section is to eliminate the measure theory from the proof of Theorem 1. In the first stage we shift attention to measures having finite support.

Fix $p, a, n$ as in the statement of Theorem 1. For each positive integer $k$ let

$$
\begin{gathered}
S_{k}=\left\{\sum_{i=1}^{k} \varepsilon_{i} 2^{-1}: \varepsilon_{i}=0,1\right\}, \\
\mu_{k}=\underset{i=1}{*}\left(p \delta_{0}+(1-p) \delta_{2^{-i}}\right),
\end{gathered}
$$

and let $\lambda_{k}$ be defined on the $n$-fold sum, $(n) S_{k}$, of $S_{k}$ with itself by the property

$$
\lambda_{k}\{x\}=2^{-k} \quad(x \in(n) S) .
$$

Eventually we will prove

TheOREM 2. Let $k$ be a positive integer and suppose that $B_{1}, B_{2}, \ldots, B_{n}$ are subsets of $S_{k}$. Then

$$
\lambda_{k}\left(B_{1}+B_{2}+\cdots+B_{n}\right) \geqslant \mu_{k}\left(B_{1}\right)^{\alpha} \mu_{k}\left(B_{2}\right)^{\alpha} \cdots \mu_{k}\left(B_{n}\right)^{\alpha} .
$$

The first task is to prove

\section{LEMMA 1. Theorem 2 implies Theorem 1.}

PROof. Let us start with another reduction by noting that it will suffice to prove Theorem 1 for closed sets. For suppose we have that limited form of the result and are given Borel subsets $E_{1}, E_{2}, \ldots, E_{n}$ of $[0,1]$. Fix $\eta>0$ and choose $\varepsilon>0$ such that $(1+\varepsilon)^{n \alpha} \leqslant 1+\eta$. By regularity of $\mu_{p}$ we may choose closed sets $F_{1}, F_{2}, \ldots, F_{n}$ such that $F_{i} \subseteq E_{i}$ and

$$
\mu_{p}\left(F_{i}\right) \geqslant(1+\varepsilon)^{-1} \mu_{p}\left(E_{i}\right), \text { for } i=1, \ldots, n \text {. }
$$

Then

$$
\begin{aligned}
\mu_{p}\left(E_{1}\right)^{\alpha} \cdots \mu_{p}\left(E_{n}\right)^{\alpha} & \leqslant(1+\varepsilon)^{n \alpha} \mu_{p}\left(F_{1}\right)^{\alpha} \cdots \mu_{p}\left(F_{n}\right)^{\alpha} \\
& \leqslant(1+\eta) \mu_{p}\left(F_{1}\right)^{\alpha} \cdots \mu_{p}\left(F_{n}\right)^{\alpha} \\
& \leqslant(1+\eta) \lambda\left(F_{1}+\cdots+F_{n}\right) \\
& \leqslant(1+\eta) \lambda\left(E_{1}+\cdots+E_{n}\right) .
\end{aligned}
$$


Since $\eta$ was an arbitrary positive number, we have verified the opening remark of the proof.

Observe next that we may write any clsoed set $F_{j}$ in $[0,1]$ as an intersection

$$
F_{i}=\bigcap_{k=1}^{\infty} F_{i}^{k}
$$

where

$$
F_{i}^{k}=B_{i}^{k}+\left[0,2^{-k}\right]
$$

A simple compactness argument shows that

$$
F_{1}+F_{2}+\cdots+F_{n}=\bigcap_{k=1}^{\infty}\left(F_{1}^{k}+F_{2}^{k}+\cdots+F_{n}^{k}\right) .
$$

We have, as $k \rightarrow \infty$,

$$
\mu_{p}\left(F_{i}^{k}\right) \rightarrow \mu_{p}\left(F_{i}\right), \quad \lambda\left(F_{1}^{k}+F_{2}^{k}+\cdots+F_{n}^{k}\right) \rightarrow \lambda\left(F_{1}+F_{2}+\cdots+F_{n}\right) .
$$

Moreover,

$$
\mu_{p}\left(F_{i}^{k}\right)=\mu_{k}\left(B_{i}^{k}\right), \quad \lambda\left(F_{1}^{k}+F_{2}^{k}+\cdots+F_{n}^{k}\right) \geqslant \lambda_{k}\left(B_{1}^{k}+B_{2}^{k}+\cdots+B_{n}^{k}\right),
$$

and so the statement of the lemma is true.

Now we must set about proving Theorem 2. We shall use an inductive argument to reduce it to a purely combinatorial result. This reduction is similar to the argument of Lemma 2.6 of [2]. That proof is unfortunately somewhat garbled so we take the opportunity to note that the sets $D_{n}^{0}, D_{n}^{1}$ defined there should be given as

$$
D_{n}^{0}=\left\{\sum_{k=1}^{n-1} \varepsilon_{k} 3^{-k}: \varepsilon_{k}=0,2\right\}, \quad D_{n}^{1}=\left\{\sum_{k=1}^{n-1} \varepsilon_{k} 3^{-k}+2 \cdot 3^{-n}: \varepsilon_{k}=0,2\right\} .
$$

Then the induction works "for the tail" in similar fashion to the following argument.

REDUCTION of Theorem 2. Suppose that we have the result of Theorem 2 for some positive integer $k$. Let $B_{1}, \ldots, B_{n}$ be subsets of $S_{k+1}$. For $j=1, \ldots, n$, we write

$$
\begin{aligned}
& B_{j, 0}=\left\{\sum_{i=1}^{k+1} \varepsilon_{j} 2^{-i} \in B_{j}: \varepsilon_{k+1}=0\right\}, \\
& B_{j, 1}=\left\{\sum_{i=1}^{k+1} \varepsilon_{i} 2^{-1} \in B_{j}: \varepsilon_{k+1}=1\right\} .
\end{aligned}
$$

Note that the sum set, $B_{1}+B_{2}+\cdots+B_{n}$, is a union of sum sets each of the form

$$
B_{1, j(1)}+B_{2, j(2)}+\cdots+B_{n, j(n)}
$$


where $j(i) \in\{0,1\}$. Let us write $\mathscr{C}$ for the collection of all those sum sets given as in (1) for which $j(1)+j(2)+\cdots+j(n)$ is even and $\mathscr{D}$ for those sum sets for which $j(1)+j(2)+\cdots+j(n)$ is odd. Then every set in $\mathscr{C}$ is disjoint from every set in $\mathscr{D}$. It follows that

$$
\begin{aligned}
& \lambda_{k+1}\left(B_{1}+B_{2}+\cdots+B_{n}\right) \\
& \quad \geqslant \max \left\{\lambda_{k+1}(C): C \in \mathscr{C}\right\}+\max \left\{\lambda_{k+1}(D): D \in \mathscr{D}\right\} .
\end{aligned}
$$

Let us use a prime to denote projection from $S_{k+1}$ to $S_{k}$. Thus we write

$$
B_{1, j(i)}^{\prime}=\left\{\sum_{m=1}^{k} \varepsilon_{m} 2^{-m}: \sum_{m=1}^{k} \varepsilon_{m} 2^{-m}+j(i) 2^{-k-1} \in B_{i, j(i)}\right\} ;
$$

also

$$
C^{\prime}=B_{1, j(1)}^{\prime}+B_{2, j(2)}^{\prime}+\cdots+B_{n, j(n)}^{\prime},
$$

provided that

$$
C=B_{1, j(1)}+B_{2, j(2)}+\cdots+B_{n, j(n)} .
$$

Using the notation introduced in (3), (4), (5), we may rewrite (2) as the inequality

$$
\begin{aligned}
& \lambda_{k+1}\left(B_{1}+B_{2}+\cdots+B_{n}\right) \\
& \quad \geqslant \frac{1}{2} \max \left\{\lambda_{k}\left(C^{\prime}\right): C \in \mathscr{C}\right\}+\frac{1}{2} \max \left\{\lambda_{k}\left(D^{\prime}\right): D \in \mathscr{D}\right\} .
\end{aligned}
$$

The inductive hypothesis will enable us to replace a term such as $\lambda_{k}\left(C^{\prime}\right)$ in (6) by an expression of the form

$$
\mu_{k}\left(B_{1, j(1)}^{\prime}\right)^{\alpha} \mu_{k}\left(B_{2, j(2)}^{\prime}\right)^{\alpha} \cdots \mu_{k}\left(B_{n, j(n)}^{\prime}\right)^{\alpha} .
$$

Now observe that

$$
\mu_{k+1}\left(B_{1, j(i)}\right)=p \mu_{k}\left(B_{i, j(i)}^{\prime}\right) \text { or }(1-p) \mu_{k}\left(B_{i, j(i)}^{\prime}\right),
$$

with the first or second alternative occurring according to whether $j(i)$ is even or odd. Moreover,

$$
\mu_{k+1}\left(B_{i, 0}\right)=x_{i} \mu_{k+1}\left(B_{i}\right), \quad \mu_{k+1}\left(B_{i, 1}\right)=\left(1-x_{i}\right) \mu_{k+1}\left(B_{i}\right),
$$

for some $0 \leqslant x_{i} \leqslant 1$. Combining (8) and (9) we find that

$$
\mu_{k}\left(B_{i, 0}^{\prime}\right)=\left(\frac{x_{i}}{p}\right) \mu_{k+1}\left(B_{i}\right), \mu_{k}\left(B_{i, 1}^{\prime}\right)=\left(\frac{1-x_{i}}{1-p}\right) \mu_{k+1}\left(B_{i}\right) .
$$

Now we may substitute (10) and (7) to see that (7) can be rewritten as an expression of the form

$$
\mu_{k+1}\left(B_{1}\right)^{\alpha} \mu_{k+1}\left(B_{2}\right)^{\alpha} \cdots \mu_{k+1}\left(B_{n}\right)^{\alpha}\left(y_{1} y_{2} \cdots y_{n}\right),
$$

where each $y_{i}$ is of the form $\left(x_{i} / p\right)^{\alpha}$ or $\left(\left(1-x_{i}\right) /(1-p)\right)^{\alpha}$, the choice being determined by the parity of the sequence $j(1), j(2), \ldots, j(n)$ which is in turn determined by $C$. Accordingly, let us relabel $y_{1} y_{2} \cdots y_{n}$ as $y(C)$ and deduce 
from (6), using the inductive hypothesis, that

$$
\begin{aligned}
\lambda_{k+1}( & \left.B_{1}+B_{2}+\cdots+B_{n}\right) \\
\geqslant & {\left[\frac{1}{2} \max j\{y(c): C \in \mathscr{C}\}+\frac{1}{2} \max \{y(D): D \in \mathscr{D}\}\right] } \\
& \cdot \mu_{k+1}\left(B_{1}\right)^{\alpha} \mu_{k+1}\left(B_{2}\right)^{\alpha} \cdots \mu_{k+1}\left(B_{n}\right)^{\alpha} .
\end{aligned}
$$

It is immediately clear that the inductive step will be accomplished once we can prove that

$$
\max \{y(C): C \in \mathscr{C}\}+\max \{y(D): D \in \mathscr{D}\} \geqslant 2 .
$$

This is the purely combinatorial theorem which we shall isolate and prove in the next section.

It remains to ground the induction by checking the case $k=1$. Each $B_{i} \subseteq$ $\left\{0, \frac{1}{2}\right\}$. If, in fact, $B_{i}=\left\{0, \frac{1}{2}\right\}$ for some $i$, then we see that $\lambda_{1}\left(B_{1}+B_{2}+\cdots+B_{n}\right)$ $\geqslant 1 \geqslant \mu_{1}\left(B_{1}\right)^{\alpha} \cdots \mu_{1}\left(B_{n}\right)^{\alpha}$. We may suppose then that each $B_{i}$ is a singleton and that $\lambda_{i}\left(B_{1}+B_{2}+\cdots+B_{n}\right)=\frac{1}{2}$. We must verify that $\left[\max (p,(1-p)]^{n \alpha} \leqslant 1 / 2\right.$. But this is the requirement that $a^{n \alpha} \geqslant 2$, and we have already chosen $\alpha=$ $\log 2 / n \log a$, so the case $k=1$ is indeed true.

\section{Combinatorial result}

It remains to prove the combinatorial theorem which corresponds to assertion (12) of the last section.

Theorem 3. Suppose that $0<p<1$ and that $n$ is a positive integer. Let $a=[\max (p,(1-p))]^{-1}$ and let $\alpha=\alpha_{n}=\log 2 / n \log a$. Suppose that $a \geqslant 2^{1 / n}$ and that $0 \leqslant x_{j} \leqslant 1$ for $i=1, \ldots, n$. Let $Y$ be the set of all products $y_{1} \cdots y_{j} \cdots y_{n}$ of $n$ terms in which the jth term $y_{j}$ satisfies one or the other of

$$
\text { (i) } y_{j}=\left(x_{j} / p\right)^{\alpha}, \quad \text { (ii) } y_{j}=\left[\left(1-x_{j}\right) /(1-p)\right]^{\alpha} \text {. }
$$

Write $Y$ as the disjoint union $Y_{0} \cup Y_{1}$, where $Y_{0}$ is the set of all products in which the second choice is made an even number of times and $Y_{1}$ is the set of products in which the second choice is made an odd number of times.

Then

$$
\max Y_{0}+\max Y_{1} \geqslant 2
$$

We have another reduction in mind.

LEMMA 2. It will suffice to prove that, for $1 \geqslant x \geqslant p$, we have

$$
G(x)=\left(\frac{x}{p}\right)^{(n-1) \alpha}\left(\left(\frac{x}{p}\right)^{\alpha}+\left[\frac{(1-x)}{(1-p)}\right]^{\alpha}\right) \geqslant 2 .
$$


Proof of LEMma. In tackling Theorem 3 we can (and do) assume without loss of generality that

$$
x_{1} \geqslant x_{2} \geqslant \cdots \geqslant x_{n} .
$$

The first case to be considered occurs when we also have $x_{n} \geqslant p$. Now the product $\left(x_{1} / p\right)^{\alpha}\left(x_{2} / p\right)^{\alpha} \cdots\left(x_{n} / p\right)^{\alpha}$ belongs to $Y_{0}$, and the product $\left(x_{1} / p\right)^{\alpha}\left(x_{2} / p\right)^{\alpha} \cdots\left(\left(x_{n-1}\right) / p\right)^{\alpha}\left(\left(1-x_{n}\right) /(1-p)\right)^{\alpha}$ belongs to $Y_{1}$. Therefore

$$
\max Y_{0}+\max Y_{1} \geqslant\left(\frac{x_{1}}{p}\right)^{\alpha} \cdots\left(\frac{x_{n-1}}{p}\right)^{\alpha}\left[\left(\frac{x_{n}}{p}\right)^{\alpha}+\left(\frac{1-x_{n}}{1-p}\right)^{\alpha}\right]
$$

Combining (13) and (14) and setting $x=x_{n}$, we obtain

$$
\max Y_{0}+\max Y_{1} \geqslant G(x),
$$

where $x \geqslant p$.

A similar argument is available when we have $p \geqslant x_{1}$, because we interchange the rôles of $p, 1-p ; x_{i}, 1-x_{i}$. This gives

$$
\max Y_{0}+\max Y_{1} \geqslant\left(\frac{1-x}{1-p}\right)^{(n-1) \alpha}\left(\left(\frac{1-x}{1-p}\right)^{\alpha}+\left(\frac{x}{p}\right)^{\alpha}\right),
$$

where $x=x_{1}$, and thus $1-x \geqslant 1-p$.

Let us now consider the case where

$$
x_{1} \geqslant \cdots \geqslant x_{k} \geqslant p \geqslant x_{k+1} \geqslant \cdots \geqslant x_{n} .
$$

There are two sub-cases. Suppose first that

$$
x_{k} / p \geqslant\left(1-x_{k+1}\right) /(1-p) .
$$

Of the two products

$$
\left(\frac{x_{1}}{p}\right)^{\alpha} \cdots\left(\frac{x_{k}}{p}\right)^{\alpha}\left(\frac{1-x_{k+1}}{1-p}\right)^{\alpha} \cdots\left(\frac{1-x_{n}}{1-p}\right)^{\alpha}
$$

and

$$
\left(\frac{x_{1}}{p}\right)^{\alpha} \cdots\left(\frac{x_{k}}{p}\right)^{\alpha}\left(\frac{x_{k+1}}{p}\right)^{\alpha}\left(\frac{1-x_{k+2}}{1-p}\right)^{\alpha} \cdots\left(\frac{1-x_{n}}{1-p}\right)^{\alpha},
$$

one belongs to $Y_{0}$ and the other to $Y_{1}$. Hence their sum gives a lower estimate for $\max Y_{0}+\max Y_{1}$. Using (17) and (18) together, we see that each term in the product labelled (19) exceeds $\left(\left(1-x_{k+1}\right) /(1-p)\right)^{\alpha}$. A similar argument shows that the product labelled (20) exceeds $\left(\left(1-x_{k+1}\right) /(1-p)\right)^{(n-1) \alpha}\left(x_{k+1} / p\right)$. Accordingly we set $x=x_{k+1}$ and obtain (16) once more (with, of course, $1-x \geqslant 1-p$ ). The other sub-case occurs when

$$
\frac{x_{k}}{p} \leqslant \frac{1-x_{k+1}}{1-p} \text {. }
$$


This time we consider the products

and

$$
\left(\frac{x_{1}}{p}\right)^{\alpha} \cdots\left(\frac{x_{k}}{p}\right)^{\alpha}\left(\frac{1-x_{k+1}}{1-p}\right)^{\alpha} \cdots\left(\frac{1-x_{n}}{1-p}\right)^{\alpha}
$$

$$
\left(\frac{x_{1}}{p}\right)^{\alpha} \cdots\left(\frac{x_{k-1}}{p}\right)^{\alpha}\left(\frac{1-x_{k}}{1-p}\right)^{\alpha} \cdots\left(\frac{1-x_{n}}{1-p}\right)^{\alpha} \text {. }
$$

We use (17) and (21) to see that the first exceeds $\left(x_{k} / p\right)^{n \alpha}$ while the second exceeds $\left(x_{k} / p\right)^{(n-1) \alpha}\left(\left(1-x_{k}\right) /(1-p)\right)$. We set $x=x_{k}$ to obtain (15) with $x \geqslant p$. This completes the proof of the lemma.

It remains to check that the inequality mentioned in the statement of Lemma 2 really does hold. This is the first occasion on which the condition $a \geqslant 2^{1 / n}$ bites, so let us recall the standing asumptions.

LEMMA 3. Suppose that $0<p<1$, that $a[\max (p,(1-p))]^{-1}$, that $n$ is a positive integer such that $a \geqslant 2^{1 / n}$ and that $\alpha=\log 2 / n \log a$.

Then

$$
F(t)=t^{n \alpha}+t^{(n-1) \alpha}\left(\frac{1-p t}{1-p}\right)^{\alpha} \geqslant 2, \quad \text { for } 1 \leqslant t \leqslant 1 / p
$$

Proof. $F(1)=2, \quad F\left(p^{-1}\right)=p^{-n \alpha} \geqslant a^{n \alpha}=2$. Accordingly it will suffice to check that $F^{\prime}(t)=0$ implies $F(t) \geqslant 2$. In fact,

$$
\begin{aligned}
F^{\prime}(t)= & n \alpha t^{n \alpha-1}+(n-1) \alpha t^{(n-1) \alpha-1}\left(\frac{1-p t}{1-p}\right)^{\alpha} \\
& -\alpha p t^{(n-1) \alpha}\left(\frac{1-p t}{1-p}\right)^{\alpha-1}(1-p)^{-1} \\
= & \alpha t^{(n-1) \alpha-1}\left[n t^{\alpha}+\left(\frac{1-p t}{1-p}\right)^{\alpha-1}\{n-1-(n-1) p t-p t\}(1-p)^{-1}\right],
\end{aligned}
$$

Thus $F^{\prime}(t)=0$ entails

$$
n t^{\alpha}=\left(\frac{1-p t}{1-p}\right)^{\alpha-1}\left(\frac{n p t-n+1}{1-p}\right) .
$$

When (22) holds, we have

$$
\begin{aligned}
F(t) & =t^{n \alpha}+t^{(n-1) \alpha} n t^{\alpha}(1-p t)(n p t-n+1)^{-1} \\
& =t^{n \alpha}(n p t-n+1)^{-1}\{n p t-n+1+n-n p t\} \\
& =p^{-n \alpha}(p t)^{n \alpha}(n p t-n+1)^{-1} .
\end{aligned}
$$


Since $p^{-n \alpha} \geqslant 2$, it will suffice to prove that

$$
(p t)^{n \alpha} \geqslant n p t-(n-1) \text {. }
$$

Let us write $s=p t$, so that (23) becomes

$$
s^{n \alpha} \geqslant n s-(n-1), \text { for } p \leqslant s \leqslant 1 .
$$

The condition $a \geqslant 2^{1 / n}$ implies that $\alpha \leqslant 1$, so to prove (24) it will be enough to prove that

$$
H(s)=s^{n}-n s+(n-1) \geqslant 0, \text { for } p \leqslant s \leqslant 1 .
$$

But $H^{\prime}(s)=n s^{n-1}-n \leqslant 0$ and $H(1)=0$, so (25) holds. this completes the proof of the lemma and hence of Theorem 3 and the results depending upon it.

\section{References}

[1] G. Brown, M. Keane, W. Moran, and C. Pearce, 'A combinatorial inequality with applications to Cantor measures,' preprint.

[2] G. Brown and W. Moran, 'Raikov systems and radicals in convolution measure algebras,' $J$. London Math. Soc. (2) 29 (1983), 531-542.

[3] G. Brown, W. Moran, and R. Tijdeman, 'Riesz products are basic measures', J. London Math. Soc. (2) 30 (1984), 105-109.

[4] E. Hewitt and K. Stromberg, Real and Abstract Analysis (Springer-Verlag, Berlin, Heidelberg, New York, 1965).

[5] D. M. Oberlin, 'The size of sum sets, II,' preprint.

Department of Pure Mathematics

University of New South Wales

Kensington, 2033, N.S.W.

Australia 